\title{
Discriminant model possibility of occurrence pubertal uterine bleeding in girls depending on the characteristics of the structure and size of the body
}

\author{
Grigoriy ChaikA*, Oksana Kucherenko
}

Vinnitsa National Medical University named after M.I. Pirogov, Vinnytsya, Ukraine

\begin{tabular}{|c|c|}
\hline ARTICLE INFO & ABSTRACT \\
\hline $\begin{array}{l}\text { Received } 07 \text { March } 2016 \\
\text { Accepted } 01 \text { April } 2016\end{array}$ & $\begin{array}{l}\text { In this paper, based on the performance characteristics of the structure and size of the } \\
\text { body, and through step discriminant analysis, we have built an accurate and highly }\end{array}$ \\
\hline $\begin{array}{l}\text { Keywords: } \\
\text { discriminant model, } \\
\text { pubertal uterine bleeding, } \\
\text { anthropometry. }\end{array}$ & $\begin{array}{l}\text { informative model of the possibility of pubertal uterine bleeding in a general population } \\
\text { of girls aged from } 14 \text { to } 18 \text { years, and as broken down into groups } 14-15 \text { and 16-18 years. } \\
\text { In the total group of girls of } 14-18 \text { years and notably in girls 16-18 years, indicators of } \\
\text { skin thickness and fat fold are consistent with such a possibility, while in girls } 14-15 \text { years } \\
\text { old, indicators of body diameter, width of distal epiphysis of long bones of the limbs and } \\
\text { encompassing body size, show correlation with pubertal uterine bleeding. The largest } \\
\text { contribution to discrimination, regardless of the age groups, is, however, the value of the } \\
\text { fat component of body weight. }\end{array}$ \\
\hline
\end{tabular}

\section{INTRODUCTION}

The main cause of puberty uterine bleeding (PUB) and adolescent period is the immaturity of the reproductive system. This manifests itself as a defective negative feedback involving the ovaries and the hypothalamic-pituitary area of the Central Nervous System (CNS) [1,2]. Herein, estrogen level increase does not lead to a reduction in the secretion of Follicle Stimulating Hormone (FSH), which in turn, induces the simultaneous stimulation of follicle growth and development. Higher than normal FSH secretion is a factor that inhibits the selection and development of the dominant follicle from a variety of maturing hollow follicles. Moreover, the lack of ovulation and subsequent production of progesterone by the luteum leads to the target organs, including the endometrium, being continuous exposed to estrogen $[3,4]$.

The somatotype conjugation of puberty, and, thus, the age of menarche, is determined by genetically programmed hormonal status, and anabolic hormones have a role in shaping the physique features [5]. This raises the question of a holistic review of standard clinical investigative/treatment practices, and suggests that these be based not only on morphometric confirmation of age and the constitutional differences of the parameters of the reproductive system in healthy and sick women and girls, but also upon

\footnotetext{
* Corresponding author

e-mail: chayka_g@mail.ru
}

the creation of models that will display the most common features of pubertal bleeding and provide an opportunity to generate reasonable predictions even at the stage of primary prevention.

The purpose of our research was to construct and to analyze discriminant models of the risk of pubertal uterine bleeding in girls, building these upon characteristic anthropo-somatotypological parameters of body.

\section{MATERIAL AND METHODS}

In this work, primary anthropo-somatotypological performance of 113 healthy girls aged from 14 to 15 years, and 121 girls aged from 16 to 18 years, are taken from the database of materials held by the Scientific and Research Center of the Vinnitsa National Medical University named after Pirogov. Moreover, within the Vinnytsya City Clinical Hospital, Vinnitsa city maternity hospital No. 1 and the Vinnytsya Central District Hospital, an anthropometric survey (built on the scheme of Bunak, a modification of that of P.P. Shaparenko [6]) was conducted. This involved 18 girls and 48 girls of similar age, who had pubertal uterine bleeding (PUB). In this, the somatotype was determined by way of the method of J. Carter and B. Heath [7], while the component composition of body weight was ascertained by the methods of J. Matiegka [8] and that of the American Institute of Nutrition [9]. 
Herein, the construction of discriminant models of risk of development pubertal bleeding was done by way a licensed statistical package - "STATISTICA 6.0".

\section{RESULTS AND DISCUSSION}

When accounting for anthropometric, somatotypological performance and component composition, the body mass discriminant function takes in $98.3 \%$ of all healthy and $94.8 \%$ of all patients with pubertal uterine bleeding that are aged between 14 to 18 years. In general, the proposed model is correct in $97.6 \%$ of all cases. Between the healthy and those afflicted with pubertal uterine bleeding (PUB), the discriminant variables are cross lower thoracic size, the fat component of body weight, the circumference of the chest at a maximum exhalation, fat thickness skin-folds (TSFF) under the shoulder blade, on the chest, on the abdomen and on the side, and the width of the distal epiphysis (WDE) hip. Still, the greatest contribution towards discriminating between the healthy and the PUB is the fat component value of body weight. In general, the totality of all variables is highly significant (Wilks statistics lambda $=0,167 ; \mathrm{F}=$ $141,5 ; \mathrm{p}<0.001)$ in regard to discriminating between the healthy and the PUB. The definition of parameter classification (Df) is given in the form of an equation. When assigned to healthy girls aged from 14 to 18 years, the Df is close to 278.4, whereas with patients afflicted with pubertal uterine bleeding (aged from 14 to 18 years), the Df value is close to 351.1 :

Df (for healthy girls - aged 14 to 18 years) = the value of the lower thoracic transverse size x 3.695 - fat component value of body weight x $14.89+$ size chest circumference at a maximum exhalation $\times 2.477$ - TSFF under the shoulder blade $x 0.116+$ TSFF on the chest $x 2,329+$ TSFF on the abdomen $\times 1.142$ - TSFF on the side $\times 2.575+$ WDE hip x 50.10-278.4;

Df (for PUB patients - aged 14 to 18 years $)=$ the value of the lower thoracic transverse size x 5.336 - fat component value of body weight $\mathrm{x} 20.41+$ size chest circumference at a maximum exhalation $\mathrm{x} 2.974$ - TSFF under the shoulder blade $x 1.519+$ TSFF on the chest $x 4.512+$ TSFF on the abdomen $\times 2.261$ - TSFF on the side x $3.369+$ WDE hip x $53.98-351.1$;

where (here and subsequently), the diameter of the body - is in cm; performance component composition of body weight - is in kilograms; encompassing body size - is in $\mathrm{cm}$; TSFF - is in mm; WDE long bones of limbs - is in cm.

When accounting for the anthropo-somatotypological rates in girls aged from 14 to 15 years, we found that the discriminant function is inclusive of $100 \%$ of all healthy and $100 \%$ of all PUB patients. Between the healthy and those afflicted with PUB, the discriminant variables are fat component of body weight, lower thoracic size, the circumference of the chest when breathing quietly and at maximum expiration, the WDE of forearm and thigh, the ectomorph component somatotype value as per Heath-Carter and the external conjugate. Still, the greatest contribution towards discriminating between the healthy and the PUB afflicted, as in the previous group, is the fat component value of body weight. In general, the totality of all variables is highly significant
(Wilks statistics lambda $=0,116 ; \mathrm{F}=97,87 ; \mathrm{p}<0.001$ ) with regard to discrimination between the healthy and those with PUB aged from 14 to 15 years. The performance of classification (Df) for this grouping is calculated as follows:

Df (for healthy girls - aged from 14 to 15 years) $=-$ fat component value of body weight x $66.61+$ value lower thoracic transverse size $\times 1.031$ - the value of the circumference of the chest when breathing quietly x $0.892+$ WDE forearm x $78.89+$ ectomorphic component size somatotype as per Heath-Carter x 31.15+ hip WDE x $126.8+$ external conjugate value $\times 9.146+$ chest circumference at maximum exhalation x 9.916-943.7;

Df (for PUB patients - aged from 14 to 15 years) $=-$ fat component value of body weight x $81,97+$ value lower thoracic transverse size $\times 2,823$ - the value of the circumference of the chest when breathing quietly x 0,624 +WDE forearm x 93,67+ ectomorphic component size somatotype as per Heath-Carter x 34,72 + hip WDE x 137,8 + external conjugate value $\mathrm{x} 11,31+$ chest circumference at maximum exhalation x 10,91 - 1197;

where (here and in the future), somatotype components - are in points; the size of the pelvis - is in $\mathrm{cm}$.

When accounting for the anthropo-somatotypological rates in girls aged from 16 to 18 , the discriminant function covers $97.5 \%$ of all healthy and $97.5 \%$ of all PUB patients. In general, the model is correct in $97.5 \%$ of all cases. Between the healthy and the PUB afflicted, the discriminant variables are the lower thoracic transverse size, fat body mass components, TSFF under the shoulder blade, and on the forearm and leg, chest circumference at a maximum exhalation, WDE of shin and dist. Spinarum. Still, the greatest contribution towards the discrimination between the healthy and the PUB afflicted, are, unlike previous groups, the value of the lower thoracic transverse size, the fat component of body weight and the chest circumference at a maximum exhalation. In general, the totality of all variables is highly significant (Wilks statistics lambda $=0,155$; $\mathrm{F}=78,49 ; \mathrm{p}<0.001$ ) with regard to discriminating between the healthy and the PUB afflicted aged 16 to 18 years. The performance of classification (Df) for this age bracket is as follows:

Df (for healthy girls - aged from 16 to 18 years) = lower value cross breast size x 5,615 - fat component value of body weight $x$ 7,093 - TSFF under the shoulder blade $\mathrm{x}$ 0,871 + value chest circumference at a maximum exhalation x 0,836 - TSFF on forearm x 2,969+WDE shin x 35,21+ dist. Spinarum x 16,85 - TSFF on the shin x 1,712 - 361,7;

Df (for PUB afflicted - aged 16 to 18 years) $=$ lower value cross breast size x 7,224 - fat component value of body weight $x$ 9,699-TSFF under the shoulder blade $\mathrm{x}$ $1,869+$ value chest circumference at a maximum exhalation x 1,376 - TSFF on forearm x 1,312+WDE shin x 30,40+ dist. Spinarum x 18,00 - TSFF on the shin x 2,407-416,4.

In determining the significance of discriminant functions, we employed $\chi^{2}$ criteria. This revealed an accurate interpretation of the obtained indices of classification between the healthy and PUB afflicted in all age groups.

In the majority of PUB patients, we observed a clear advance (acceleration) in height and body weight, but body mass index was deemed relatively insufficient (except 
in patients aged 11-18 years). We also saw that excessive acceleration of biological maturation at the beginning of puberty varies due to slower development in the older age groups [4].

Previous researchers $[5,10]$ revealed a kind of dependence in terms of maturation of the female body, by body type. Earlier timing of menarche appearance, and, in fact, early sexual development, are determined in healthy girls as being hypersthenic, with good development of muscles and skeleton regardless of degree of severity of fat deposits. Moreover, later periods are, with regard to the asthenic body type, characteristic [5].

In adolescents, menstruation may be abundant, especially against anovulation, which is often accompanied by hyperplasia of the endometrium, and the lack of anovulatory bleeding predetermines the antiestrogen that induces ovulation [1]. Fat cells convert androgens into estrogens, which, in turn, increase inadequate secretion of luteinizing hormone due to a positive feedback mechanism, subsequently inducing endometrial hyperplasia followed by its detachment and by massive metrorrhagia. Hence, the more pronounced the fat component of body weight, the more this sequence of events is expressed [11,12].

Given the above, it may be assumed that constitutional indicators that characterize as physical development, and the degree of fatty tissue - fat thickness skin-folds (TSFF) and fat body mass play a differentiating role in the separation of the healthy and those afflicted with PUB. This, which is confirmed by the results of our study.

Thus, based on anthropometric, somatotypological and component composition indicators of body weight in girls aged from 14 to 18 years, and in the individual age groupings of 14 to 15 and from 16 to 18 years, we have developed a highly informative discriminant model for predicting the risk of pubertal uterine bleeding (PUB). The obtained results make it possible to carry out early preventive measures to prevent pubertal uterine bleeding, and to prove the expediency of the data obtained in clinical practice.

\section{CONCLUSION}

1. Our discriminant model, which is based upon anthropometric and somatotypological indices, as well as component composition and body mass, allows medical practitioners to predict the possibility of pubertal uterine bleeding (PUB) in girls aged 14-18 years (correct in 97,6 $\%$ of cases; Wilks lambda statistics $=0,167 ; \mathrm{p}<0,001)$, and particularly in girls of 14-15 years of age (correct $100 \%$ of cases, statistics Wilks lambda $=0.116, \mathrm{p}<0.001)$, as well as in girls aged 16-18 years (correct в 97,5\% of cases; Wilks lambda statistics $=0,155 ; \mathrm{p}<0,001)$.

2. The derived models incorporated: in the total group of girls 14-18 years, thickness of skin and fat folds (50.0\%); in girls aged 14-15 years - the diameter of the body, the width of distal epiphysis of the long limb bones and the encompassing body size (by $25.0 \%$ ); in girls of $16-18$ years - thickness of skin and fat folds $(37.5 \%)$ and the diameter of the body $(25.0 \%)$. The largest contribution to discrimination regardless of the age groups, is the fat component value of body weight.

\section{REFERENCES}

1. Kokolina V.F. Uterine bleeding in puberty. Manual for physicians. M.: "Adamant". 40, 2011.

2. Başaran H.O. et al. Dysfunctional uterine bleeding in adolescent girls and evaluation of their response to treatment. Turk. Journal Pediatr. 55, 2, 2013.

3. Chan S. et al. Menstrual problems and health-seeking behaviour in Hong Kong Chinese girls. Hong Kong Medical Journal. 15, 2009.

4. Kulakov V.I., Savelieva G.M., Manukhin I.B. Gynecology: national leadership. "GEOTAR - Media". 1088, 2009.

5. Onyiriuka A.N., Egbagbe E.E. Anthropometry and menarcheal status of adolescent nigerian urban senior secondary school girls. International Journal of Endocrinology and Metabolism. 11, 2, 2013.

6. Shaparenko P.P. Anthropometry. Vinnitsa, 71, 2000.

7. Carter J.E.L. The Heath-Carter anthropometric somatotype. Instruction Manual. Surrey, Canada: TeP and Rosscraft, 154, 2002.

8. Nikolic S. et al. Analysis of body mass components in national club football players in republic of Macedonia. Medicine Archives. 68, 3, 2014.

9. Piettra Moura Galvão Pereira et al. Development and validation of anthropometric equations to estimate appendicular muscle mass in elderly women. Pereira et al. Nutrition Journal. 12, 92, 2013.

10. Arpita Mandal (Nandi), Das Chaudhuri A.B. Anthropometric Hormonal Correlation: An Overview. Kamla-Raj. Life Science Journal. 2, 2, 2010.

11. Horstman A.M. et al. The role of androgens and estrogens on healthy aging and longevity. The journals of gerontology. Series A, Biological sciences. 67, 11, 2012.

12. Harold Burrows. Biological Actions of Sex Hormones. Cambridge University Press. 2013. 